\title{
Diffuse Supernova Neutrino Background Search at Super-Kamiokande
}

\section{S. El Hedri, ${ }^{a, *}$ Y. Ashida ${ }^{b}$ and A. Giampaolo ${ }^{c}$ on behalf of the Super-Kamiokande Collaboration \\ (a complete list of authors can be found at the end of the proceedings)}

${ }^{a}$ Ecole Polytechnique, IN2P3-CNRS, Laboratoire Leprince-Ringuet, F-91120 Palaiseau, France

${ }^{b}$ Kyoto University, Department of Physics

Kyoto, Kyoto 606-8502, Japan ${ }^{\dagger}$

E-mail: elhedri@llr.in2p3.fr, assy@scphys.kyoto-u.ac.jp,

giampaolo@llr.in2p3.fr

We present the results of a search for the Diffuse Supernova Neutrino Background (DSNB) at Super-Kamiokande (SK) that incorporates $22.5 \times 2970$ kton.days of data from its fourth datataking phase. Two analyses, with different energy regimes covering the 9.3-81.3 MeV antineutrino energy range, have been developed based on previous SK searches, with substantially improved background modeling and data reduction techniques. Moreover, a combined analysis of nearly 20 years of SK data (1996-2018) has been performed, with a 90\% C.L. sensitivity to the DSNB flux comparable to various DSNB predictions. No significant evidence for a DSNB signal has been observed, and both analyses set world-leading limits on the DSNB flux that can reach about $2.7 \bar{v}_{e} \cdot \mathrm{cm}^{-2} \cdot \mathrm{s}^{-1}$ for a wide range of models.

$37^{\text {th }}$ International Cosmic Ray Conference (ICRC 2021)

July 12 th - 23rd, 2021

Online - Berlin, Germany

\footnotetext{
${ }^{*}$ Presenter

${ }^{\dagger}$ Until end of year 2019
} 


\section{Introduction}

The Diffuse Supernova Neutrino Background (DSNB) formed by the accumulation of neutrinos from distant supernovae, carries invaluable information about the aggregate properties of supernovae and the history of the Universe. The associated signal is however particularly difficult to detect at neutrino experiments, and upper bounds on the DSNB rate have been set by a wide array of experiments. Most of these experiments target the Inverse Beta Decays (IBDs) of $O(10) \mathrm{MeV}$ electron antineutrinos. An IBD produces a positron, associated with a prompt, easily identifiable signature, and a neutron, that can be detected through the $2.2 \mathrm{MeV}$ photon produced by its capture on a proton. To date, the most stringent limits on the DSNB flux have been set by the Super-Kamiokande experiment (SK), a water Cherenkov detector in the Kamioka mine (Japan).

In these proceedings, we describe a new DSNB search at Super-Kamiokande, that takes advantage of the $22.5 \times 2970$ kton.day of data taken between 2008 and 2018. During this datataking phase, dubbed SK-IV, the trigger system has been adapted to allow for neutron capture identification. Hence, we present two analyses, covering the 9.3 to $81.3 \mathrm{MeV}$ neutrino energy range. In $9.3-31.3 \mathrm{MeV}$, we derive differential upper limits on the $\bar{v}_{e}$ flux independently from the DSNB model, following the strategy outlined in Ref. [1] using the $22.5 \times 2970$ kton.days of SK-IV data. Conversely, in 17.3-81.3 MeV, we constrain DSNB models using spectral fits and combine our constraints with the ones from Ref. [2], derived at the previous SK phases, thus using almost 20 years of data. In what follows, we describe the modeling of the signal and background processes, the different reduction steps, and the specificities of the final analyses.

\section{Signal and background modeling}

At SK, an IBD of a DSNB antineutrino will be associated with the prompt emission of Cherenkov light by the positron, followed by a weak delayed signal from the subsequent neutron capture. In this analysis, we consider positron spectra predicted by various discrete DSNB models, as well as the continuous parameterizations of the DSNB flux described in Ref. [4], that assumes a blackbody neutrino emission spectrum. We simulate the corresponding IBD signatures in the detector using a simulation that reproduces the properties of the water in SK, as well as models the PMT properties and electronic response as a function of time. To model the background associated with the weak neutron capture signal — noise from photomultipliers (PMTs) and low energy radioactive decays - we collect noise samples using a random trigger and add them to the simulated neutron capture events.

In addition to the signal we also need to model a wide variety of background sources. Elastic interactions of solar neutrinos with electrons will provide a sizable background up to $20 \mathrm{MeV}$, that will however be easily suppressed using neutron tagging. Electron antineutrinos produced in nearby reactors will lead to an irreducible background that will dominate over the DSNB signal up to reconstructed positron energies of about $10 \mathrm{MeV}$. We model them using the simulation described above, as well as the SKReact code ${ }^{1}$, developed for the SK reactor neutrino analysis. In most of the analysis window, the dominant backgrounds will arise from the spallation of cosmic ray muons

\footnotetext{
1https://github.com/Goldie643/SKReact
} 
and from atmospheric neutrino interactions. We model spallation backgrounds using data-driven techniques, as discussed in Ref. [2], and atmospheric backgrounds using a MC simulation.

\section{Data reduction}

We apply reduction cuts to the data taken by the Super High Energy (SHE) trigger in SKIV, whose threshold lies around 7.5 and $9.5 \mathrm{MeV}$ electron-equivalent kinetic energy ${ }^{2}$ for most of the SK-IV period ${ }^{3}$. After applying event reconstruction on the prompt signal, followed by mild noise reduction and event quality cuts, we process the data using three reduction steps: spallation reduction, positron candidate selection, and neutron tagging.

Spallation reduction: Cosmic muons traveling through water can interact with oxygen and induce the production of radioactive isotopes, whose decays can mimic signal events. Moreover, while most isotope decays do not lead to neutron emission, a few isotopes, in particular ${ }^{9} \mathrm{Li}$, undergo a $\beta+n$ decay that closely resembles an IBD. For this analysis, we therefore developed a set of cuts that allow to reduce spallation backgrounds without relying on neutron identification. We notably follow the data-driven strategies developed in Ref. [2] and Ref. [1], that investigated correlations between DSNB event candidates and preceding muons using a likelihood analysis. Here, we improved these strategies in two ways. First, we introduced preselection cuts that allow to remove about half of the spallation backgrounds while only losing about $2 \%$ of the signal. These cuts remove isotope decays clustered in time and space, and events that are observed close to neutrons associated with muon-induced hadronic showers. Second, we refine the likelihood analysis described in Ref. [2] by accounting for possible correlations between observables. These techniques allow for an up to $60 \%$ increase in signal efficiency compared to the reduction steps used for the previous SK-IV DSNB analysis [1].

DSNB positron candidate selection: These cuts are aimed at radioactivity events not removed by noise reduction cuts, as well as at muons, pions, and gamma rays produced by atmospheric neutrino interactions. Here, we use the same cuts as the ones described in the SK analysis from Ref. [2]. These cuts are based on the events' distance to the walls of SK's inner detector, their directions, and their Cherenkov light patterns. Overall, these cuts allow to remove about two thirds of the atmospheric backgrounds while keeping $\sim 85 \%$ of the signal.

Neutron tagging: Updates in the trigger system performed right before SK-IV allowed to save most neutrons captures following SHE-triggered events, for the first time at SK. In this analysis we exploited this new capability to fully characterize IBD processes, using a boosted decision tree to identify neutron captures in coincidence with $O(10) \mathrm{MeV}$ events. A detailed description of the neutron tagging procedure and the systematic uncertainty evaluation can be found in [cite A. Giampaolo's proceedings]. For a 10\% signal efficiency, this algorithm allows to reduce neutronless backgrounds by a factor of 10000 , a factor of 7 improvement compared to the algorithm described in Ref. [3] and an order of magnitude improvement compared to the previous SK analysis from

\footnotetext{
${ }^{2}$ This observable is defined as the reconstructed energy minus the electron mass. For an electron or a positron, it thus corresponds to the reconstructed kinetic energy.

${ }^{3}$ From 2008 to 2010 , the threshold was $9.5 \mathrm{MeV}$
} 


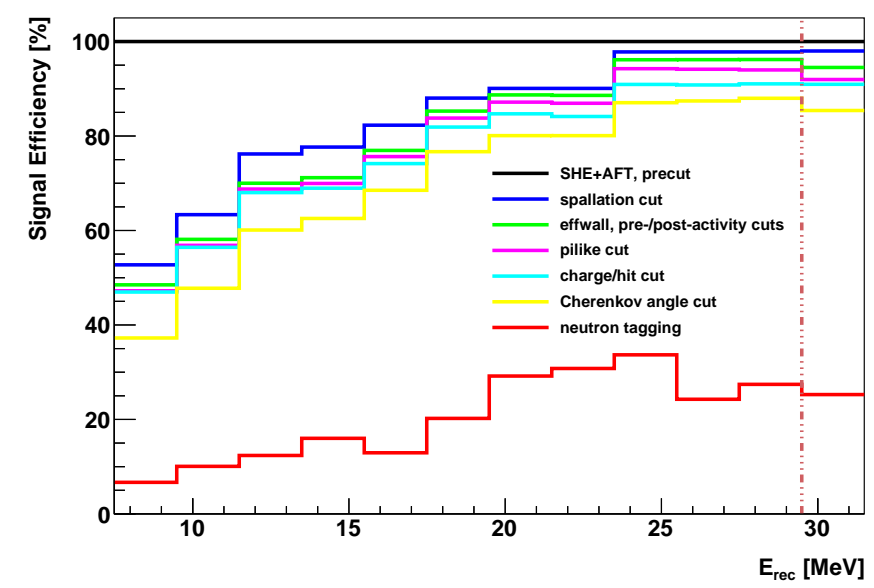

Figure 1: Signal efficiencies as a function of energy for the model-independent and spectral analyses. The effwall, pilike, charge/hit, and Cherenkov angle cuts are part of the DSNB positron candidate selection procedure described in Ref. [2].

Ref. [1]. For the analyses presented here, we will consider neutron tagging efficiencies and mistag rates ranging from 17 to $35 \%$ and 0.07 to $10 \%$ respectively.

As discussed in Section 1, we will present two DSNB analyses: one differential limit on the DSNB flux for neutrino energies ranging from $9.3 \mathrm{MeV}$ to $31.3 \mathrm{MeV}$, and a spectral analysis in the 17.3-81.3 MeV range. To facilitate the interpretation of the data, we will adopt common cuts for both analyses, maximizing the exclusion significance in different energy bins. The corresponding efficiencies as a function of energy are shown in figure 1 and vary between 6 and 33\%. This low efficiency is primarily due to neutron tagging. For the spectral analysis, in addition to events with one neutron in the 15.5-79.5 MeV range, we hence consider events failing the neutron tagging cut if their energies are larger than $19.5 \mathrm{MeV}$. Note that previous SK spectral analyses from Ref. [1] considered all events down to $15.5 \mathrm{MeV}$; however, in the absence of tight upper bounds on spallation backgrounds in the 15.5-19.5 MeV region for SK-IV, we decided to leave this energy range for further study.

\section{DSNB Model-independent Analysis}

This section describes a model-independent search for DSNB neutrinos, using the $22.5 \times 2970$ kton.days of SK-IV data. For this analysis we consider a 7.5-29.5 MeV electron-equivalent kinetic energy window divided into $2 \mathrm{MeV}$ bins, and compare the background prediction to the observations in each bin. This search draws from a former SK-IV analysis presented in Ref. [1], with significantly improved background modeling. In addition to so-called "accidental backgrounds", for which a prompt SHE event is associated with a fake IBD neutron, we indeed consider $\beta+n$ decays of spallation-produced ${ }^{9} \mathrm{Li}$, and atmospheric neutrino interactions.

We divide atmospheric neutrino interactions into two categories: neutral current quasi-elastic (NCQE) interactions, that dominate at low energies, and other types of interactions, that mostly contribute at higher energies. We evaluate NCQE backgrounds using a MC simulation, and estimate 
the associated systematic uncertainties using T2K measurements from Ref. [6]. Conversely, nonNCQE backgrounds, mainly composed of electrons from invisible muon and pion decays, are evaluated using the $29.5-79.5 \mathrm{MeV}$ region as a sideband. Finally, we evaluate the uncertainty on the ${ }^{9} \mathrm{Li}$ rate using samples of muons and DSNB candidate events, reweighted to account for the ${ }^{9} \mathrm{Li}$ half-life. NCQE and ${ }^{9} \mathrm{Li}$ backgrounds are the leading source of systematic uncertainty in this analysis, with uncertainties of around $60 \%$. Since accidental backgrounds can be directly estimated from data, however, the total systematic uncertainty in the analysis window ranges from 10 to $20 \%$.

The observed and predicted background spectra after the different reduction steps are shown in figure 2, as well as an example of a DSNB spectrum, using the $6 \mathrm{MeV}$ blackbody emission model described in Ref. [4]. This figure also shows the corresponding expected and observed 90\% C.L. upper limits on the DSNB flux as a function of energy. These limits are the world's most sensitive over the whole analysis range.
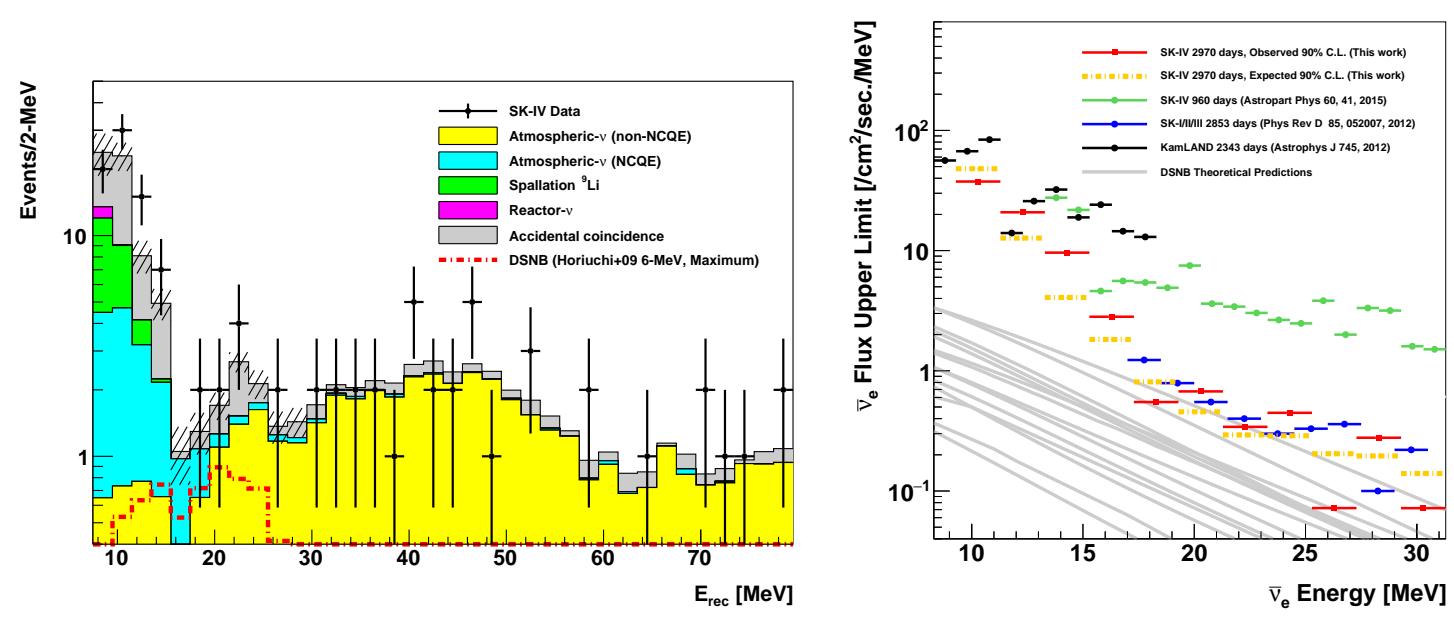

Figure 2: Left: Reconstructed energy spectra after data reduction for the background expectation and observation including the signal window and the side-band region. The shaded bars represent the systematic uncertainties. The red dashed line represents a DSNB signal expectation from the Horiuchi+09 model [4] shown only for the signal window. Right: upper limits on the extraterrestrial electron antineutrino flux from the present work, in comparison with previous published results from SK [1, 2] and KamLAND [7] and theoretical predictions from Refs [2, 8-13] (in gray).

\section{Spectral fitting}

For this search, we fit the energy spectra of the DSNB signal and of the atmosheric backgrounds in the 15.5-79.5 MeV energy range. This analysis is based on the search performed in Ref. [2], which constrained atmospheric backgrounds using sidebands for events with low and high Cherenkov angles. Here, in addition to these sidebands, we exploit the neutron tagging capabilities of SK-IV by defining separate regions for events with exactly one neutron and for events with zero or $>1$ neutron and energies larger than $19.5 \mathrm{MeV}$. Our final analysis thus considers 6 different regions: two signal regions and four sidebands.

Following the strategy described in Ref. [2], we consider four categories of atmospheric backgrounds, with distinct spectral shapes. First, electrons from invisible muon and pion decays 
will dominate in the signal regions in the $20-50 \mathrm{MeV}$ range. Their well-characterized Michel spectrum can be modeled using cosmic muon samples. Above $50 \mathrm{MeV}$, the main background in the signal regions will arise from CC interactions of electron neutrinos. Below $20 \mathrm{MeV}$, NCQE interactions will contaminate the signal regions at low energies, and will largely dominate in the high Cherenkov angle sidebands. Finally, charged current (CC) interactions producing muons and pions will be mostly found at low Cherenkov angles and will be shown to negligibly contribute to the background in the signal regions. The spectral shapes for these last three background categories are evaluated using MC simulations.

Once the spectral shapes for the signal and the all background categories are determined, we find the numbers of signal and background events using an extended maximum likelihood analysis. Specifically, for $N_{\text {events }}$ observed events with energies $\left\{E_{1}, \ldots, E_{N_{\text {events }}}\right\}$ we maximize the following likelihood:

$$
\mathcal{L}\left(\left\{N_{j}\right\}\right)=e^{-\sum_{j=1}^{5} N_{j}} \prod_{i=1}^{N_{\text {events }}} \sum_{j=0}^{4} N_{j} \operatorname{PDF}_{j}^{(r)}\left(E_{i}\right) .
$$

where $j=0, \ldots, 4$ refers to the signal and the four atmospheric background categories described above. Then $\left\{N_{j}\right\}$ designates the numbers of events for the signal and the different background types, across all six Cherenkov angle and neutron regions. For each event, $\operatorname{PDF}_{j}^{(r)}\left(E_{i}\right)$ is the PDF for the signal or background category $j$ in the region $r$. As discusss in Ref. [2], we incorporate systematic uncertainties on the background spectral shapes and the relative normalization of the PDFs in the different regions by distorting the likelihood function. Systematic uncertainties, however, impact the final limits on the DSNB flux by only a few percents.

The observations and best-fit predictions in the six Cherenkov angle and neutron regions are shown in Fig. 3 for the $6 \mathrm{MeV}$ blackbody model from Ref. [4]. The upper limits on the DSNB flux for SK-IV alone lie around $3.6 \bar{v}_{e} . \mathrm{cm}^{2}$.s for the DSNB models considered here. In the light of the weak impact of the systematic uncertainties, however, results from this analysis can be readily combined with the results from Ref. [2], thus taking advantage of almost 20 years of data. The associated best-fit values, sensitivities, and 90\% C.L. flux limits on different DSNB models [2, 4] are shown in Fig. 4. This analysis is currently improves over the sensitivity from Ref. [2] by close to $40 \%$. The associated upper limits on the DSNB flux are the tightest to date, around $2.7 \bar{v}_{e} \cdot \mathrm{cm}^{2} . \mathrm{s}$, up to $20 \%$ tighter than in the previous SK analysis.

\section{Conclusion}

In these proceedings we described two DSNB searches that used the $22.5 \times 2970$ kton.days of data taken at SK between 2008 and 2018. One of these analyses, relying on spectral fits, has notably been combined with results from previous SK phases, allowing to use nearly 20 years of data-taking, and reach a world leading sensitivity on par with the predictions from several realistic DSNB models. So far, no evidence for a DSNB signal has been found and upper limits on its flux have been set in the 9.3-81.3 MeV neutrino energy range. Finally, by exploiting the neutron tagging capabilities of SK-IV, these analyses lay the groundwork for future searches at Super-Kamiokande Gadolinium, whose prospects for a DSNB discovery are particularly promising. 

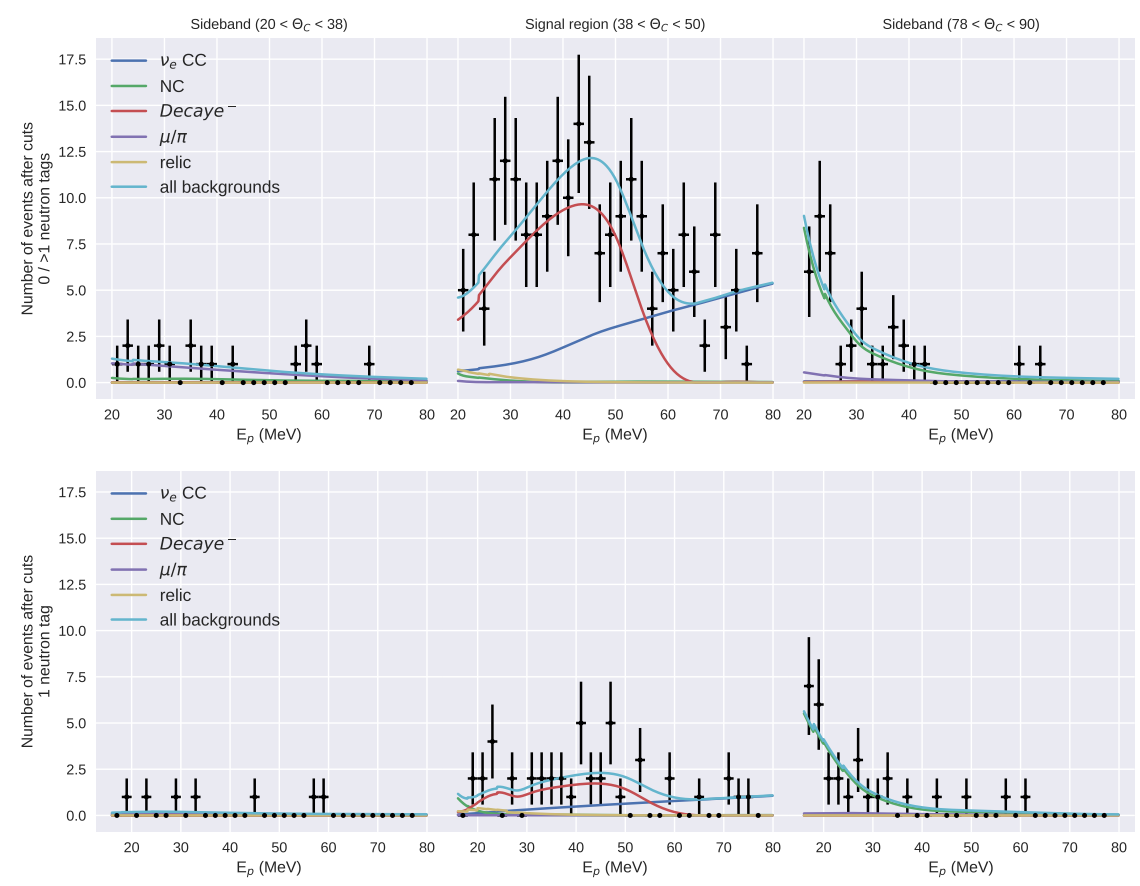

Figure 3: Observation and best-fit signal and background predictions for the spectral analysis at SuperKamiokande IV. Top: events with zero or $>1$ tagged neutron(s). Bottom: events with exactly one tagged neutron. From left to right: low, medium, and high Cherenkov angle region. The DSNB model assumed here is the $6 \mathrm{MeV}$ blackbody model from Ref. [4].

\section{References}

[1] H. Zhang et al. [Super-Kamiokande], Astropart. Phys. 60, 41-46 (2015) doi:10.1016/j.astropartphys.2014.05.004 [arXiv:1311.3738 [hep-ex]].

[2] K. Bays et al. [Super-Kamiokande], Phys. Rev. D 85, 052007 (2012) doi:10.1103/PhysRevD.85.052007 [arXiv:1111.5031 [hep-ex]].

[3] Y. Zhang et al. [Super-Kamiokande], Phys. Rev. D 93, no.1, 012004 (2016) doi:10.1103/PhysRevD.93.012004 [arXiv:1509.08168 [hep-ex]].

[4] S. Horiuchi, J. F. Beacom and E. Dwek, Phys. Rev. D 79, 083013 (2009) doi:10.1103/PhysRevD.79.083013 [arXiv:0812.3157 [astro-ph]].

[5] Y. Hayato, Acta Phys. Polon. B 40, 2477-2489 (2009)

[6] K. Abe et al. [T2K], Phys. Rev. D 100, no.11, 112009 (2019) doi:10.1103/PhysRevD.100.112009 [arXiv:1910.09439 [hep-ex]].

[7] A. Gando et al. [KamLAND], Astrophys. J. 745, 193 (2012) doi:10.1088/0004-637X/745/2/193 [arXiv:1105.3516 [astro-ph.HE]]. 

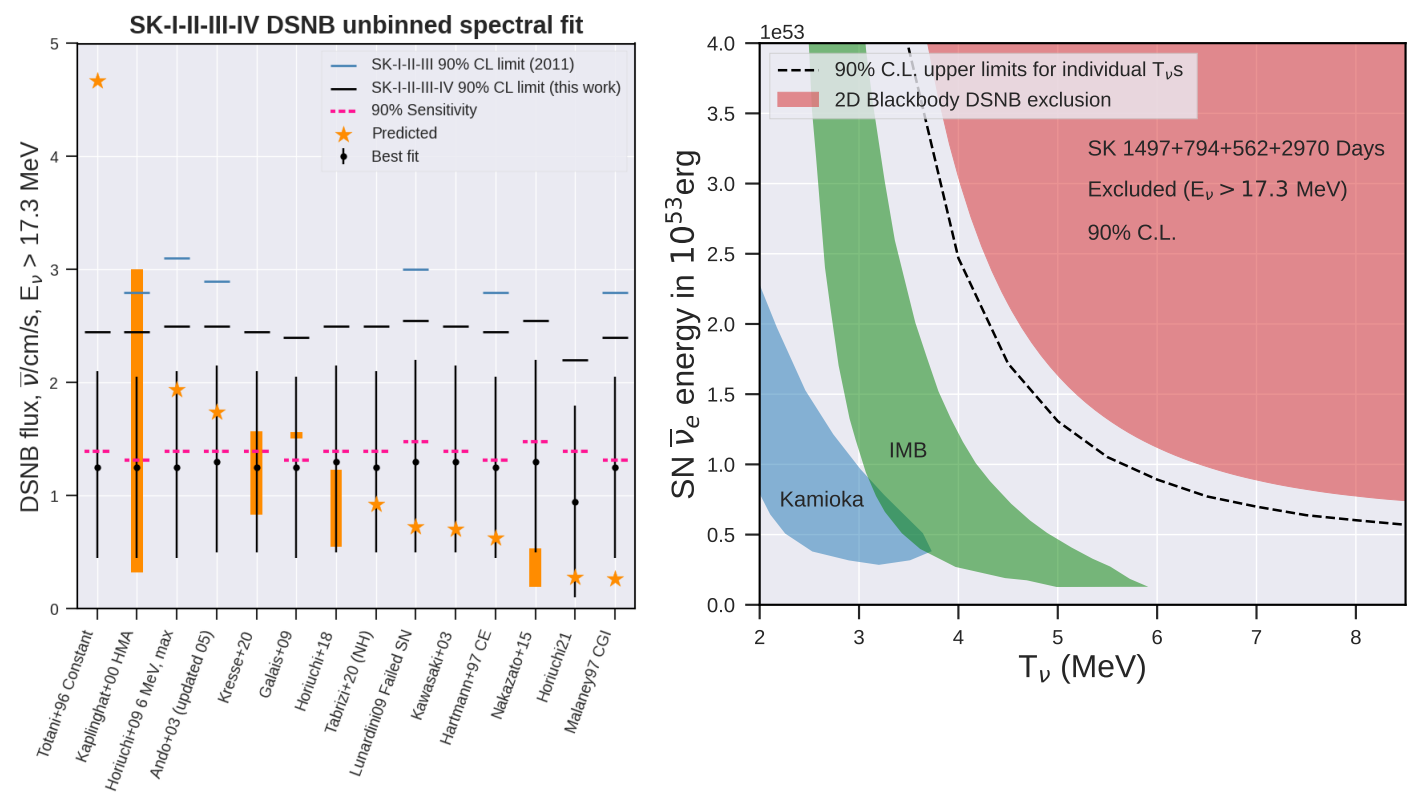

Figure 4: Left: $90 \%$ confidence limits on the DSNB flux, best-fit fluxes, and sensitivities for the combined SK analysis for a range of DSNB models [2, 8-13]. Also shown are the limits from the previous analysis from Ref. [1] and the predicted fluxes for each model. For the models considered in both analyses the limits improve by up to $20 \%$. Right: Two-dimensional confidence contours on the total supernova $\bar{v}_{e}$ energy and the effective neutrino temperature for the blackbody emission models described in Ref. [2]. To guide the eye, we also show the one-dimensional confidence limit for individual models, and the regions allowed for SN1987A [14].

[8] D. Kresse, T. Ertl and H. T. Janka, Astrophys. J. 909, no.2, 169 (2021) doi:10.3847/15384357/abd54e [arXiv:2010.04728 [astro-ph.HE]].

[9] S. Galais, J. Kneller, C. Volpe and J. Gava, Phys. Rev. D 81, 053002 (2010) doi:10.1103/PhysRevD.81.053002 [arXiv:0906.5294 [hep-ph]].

[10] S. Horiuchi, T. Kinugawa, T. Takiwaki, K. Takahashi and K. Kotake, Phys. Rev. D 103, no.4, 043003 (2021) doi:10.1103/PhysRevD.103.043003 [arXiv:2012.08524 [astro-ph.HE]].

[11] S. Horiuchi, K. Sumiyoshi, K. Nakamura, T. Fischer, A. Summa, T. Takiwaki, H. T. Janka and K. Kotake, Mon. Not. Roy. Astron. Soc. 475, no.1, 1363-1374 (2018) doi:10.1093/mnras/stx3271 [arXiv:1709.06567 [astro-ph.HE]].

[12] Z. Tabrizi and S. Horiuchi, JCAP 05, 011 (2021) doi:10.1088/1475-7516/2021/05/011 [arXiv:2011.10933 [hep-ph]].

[13] K. Nakazato, E. Mochida, Y. Niino and H. Suzuki, Astrophys. J. 804, no.1, 75 (2015) doi:10.1088/0004-637X/804/1/75 [arXiv:1503.01236 [astro-ph.HE]].

[14] B. Jegerlehner, F. Neubig and G. Raffelt, Phys. Rev. D 54, 1194-1203 (1996) doi:10.1103/PhysRevD.54.1194 [arXiv:astro-ph/9601111 [astro-ph]]. 


\section{Full Authors List: Super-Kamiokande Collaboration}

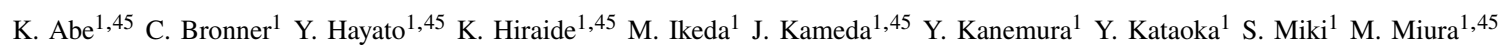
S. Moriyama ${ }^{1,45}$ Y. Nagao ${ }^{1}$ M. Nakahata ${ }^{1,45}$ S. Nakayama ${ }^{1,45}$ K. Okamoto $^{1}$ G. Pronost ${ }^{1}$ H. Sekiya ${ }^{1,45}$ M. Shiozawa ${ }^{1,45}$ Y. Sonoda $^{1}$ Y. Suzuki ${ }^{1}$ A. Takeda ${ }^{1,45}$ Y. Takemoto ${ }^{1}$ A. Takenaka ${ }^{1}$ H. Tanaka ${ }^{1}$ S. Watanabe ${ }^{1}$ T. Yano ${ }^{1}$ S. Han ${ }^{2}$ T. Kajita ${ }^{2,45}$ K. Okumura ${ }^{2,45}$ T. Tashiro ${ }^{2}$ J. Xia ${ }^{2}$ G. D. Megias ${ }^{3}$ L. Labarga ${ }^{4}$ Ll. Marti ${ }^{4}$ B. Zaldivar ${ }^{4}$ B. W. Pointon ${ }^{6,49}$ E. Kearns ${ }^{5,45}$ J. L. Raaf ${ }^{5}$ L. Wan ${ }^{5}$ T. Wester ${ }^{5}$

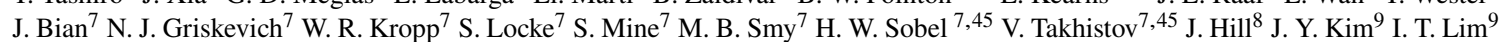
R. G. Park ${ }^{9}$ B. Bodur ${ }^{10}$ K. Scholberg ${ }^{10}$ C. W. Walter ${ }^{10,45}$ L. Bernard ${ }^{11}$ A. Coffani ${ }^{11}$ O. Drapier ${ }^{11}$ S. El Hedri ${ }^{11}$ A. Giampaolo ${ }^{11}$ M. Gonin ${ }^{11}$ Th. A. Mueller ${ }^{11}$ P. Paganini ${ }^{11}$ B. Quilain ${ }^{11}$ T. Ishizuka ${ }^{12}$ T. Nakamura ${ }^{13}$ J. S. Jang ${ }^{14}$ J. G. Learned ${ }^{15} \mathrm{~S} \mathrm{Cao}^{16}$ L. H. V. Anthony ${ }^{17}$ D. Martin ${ }^{17}$ M. Scott ${ }^{17}$ A. A. Sztuc ${ }^{17}$ Y. Uchida ${ }^{17}$ V. Berardi ${ }^{18}$ M. G. Catanesi ${ }^{18}$ E. Radicioni ${ }^{18}$ N. F. Calabria ${ }^{19}$ L. N. Machado ${ }^{19}$ G. De Rosa ${ }^{19}$ G. Collazuol ${ }^{20}$ F. $\operatorname{Iacob}^{20}$ M. Lamoureux ${ }^{20}$ M. Mattiazzi ${ }^{20}$ N. Ospina ${ }^{20}$ L. Ludovici ${ }^{21}$ Y. Maekawa $^{22}$ Y. Nishimura ${ }^{22}$ M. Friend ${ }^{23}$ T. Hasegawa ${ }^{23}$ T. Ishida ${ }^{23}$ T. Kobayashi ${ }^{23}$ M. Jakkapu ${ }^{23}$ T. Matsubara ${ }^{23}$ T. Nakadaira ${ }^{23}$ K. Nakamura ${ }^{23,45}$ Y. Oyama ${ }^{23}$ K. Sakashita ${ }^{23}$ T. Sekiguchi ${ }^{23}$ T. Tsukamoto ${ }^{23}$ T. Boschi ${ }^{24}$ F. Di Lodovico ${ }^{24}$ J. Gao ${ }^{24}$ J. Migenda ${ }^{24}$ M. Taani ${ }^{24}$ S. Zsoldos ${ }^{24}$ Y. Nakano ${ }^{25}$ H. Ozaki $^{25}$ A. T. Suzuki ${ }^{25}$ Y. Takeuchi ${ }^{25,45}$ S. Yamamoto ${ }^{25}$ Y. Kotsar ${ }^{25}$ A. Ali ${ }^{26}$ J. Feng $^{26}$ T. Kikawa ${ }^{26}$ M. Mori $^{26}$ T. Nakaya $a^{26,45}$ R. A. Wendell ${ }^{26,45}$ K. Yasutome ${ }^{26}$ P. Fernandez ${ }^{28}$ N. McCauley ${ }^{28}$ P. Mehta ${ }^{28}$ K. M. Tsui ${ }^{27}$ Y. Fukuda ${ }^{28}$ Y. Itow ${ }^{29,30}$ H. Menjo ${ }^{29}$ K. Sato ${ }^{29}$ J. Lagoda ${ }^{31}$ S. M. Lakshmi ${ }^{31}$ P. Mijakowski ${ }^{31}$ J. Zalipska ${ }^{31}$ J. Jiang ${ }^{32}$ C. K. Jung ${ }^{32}$ C. Vilela ${ }^{32}$ M. J. Wilking ${ }^{32}$ C. Yanagisawa ${ }^{32}$ M. Harada ${ }^{33}$ H. Ishino ${ }^{33}$ S. Ito ${ }^{33}$ H. Kitagawa ${ }^{33}$ Y. Koshio ${ }^{33,45}$ W. Ma ${ }^{33}$ N. Piplani ${ }^{33}$ S. Sakai ${ }^{33}$ G. Barr ${ }^{34}$ D. Barrow ${ }^{34}$ L. Cook ${ }^{34,45}$ A. Goldsack ${ }^{34,45}$ S. Samani ${ }^{34}$ D. Wark ${ }^{34,39}$ F. Nova ${ }^{35}$ J. Y. Yang ${ }^{36}$ S. J. Jenkins ${ }^{37}$ M. Malek ${ }^{37}$ J. M. McElwee ${ }^{37}$ O. Stone ${ }^{37}$

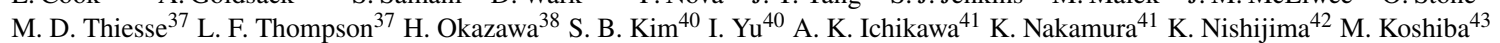
K. Iwamoto ${ }^{44}$ Y. Nakajima ${ }^{44}$ N. Ogawa ${ }^{44}$ M. Yokoyama $a^{44,45}$ K. Martens ${ }^{45}$ M. R. Vagins ${ }^{45,7}$ M. Kuze ${ }^{46}$ S. Izumiyama ${ }^{46}$ M. Inomoto ${ }^{47}$ M. Ishitsuka ${ }^{47}$ H. Ito ${ }^{47}$ T. Kinoshita ${ }^{47}$ R. Matsumoto ${ }^{47}$ M. Shinoki ${ }^{47}$ T. Suganuma ${ }^{47}$ J. F. Martin ${ }^{48}$ H. A. Tanaka ${ }^{48}$ T. Towstego $0^{48}$ R. Akutsu ${ }^{49}$ P. de Perio ${ }^{49}$ M. Hartz ${ }^{49}$ A. Konaka ${ }^{49}$ N. W. Prouse ${ }^{49}$ S. Chen $^{50}$ B. D. Xu ${ }^{50}$ M. Posiadala-Zezula ${ }^{51}$ D. Hadley ${ }^{52}$

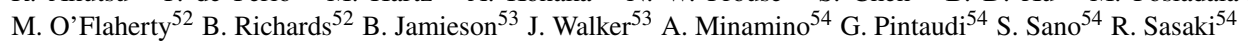

${ }^{1}$ Kamioka Observatory, Institute for Cosmic Ray Research, University of Tokyo, Kamioka, Gifu 506-1205, Japan.

${ }^{2}$ Research Center for Cosmic Neutrinos, Institute for Cosmic Ray Research, University of Tokyo, Kashiwa, Chiba 277-8582, Japan.

${ }^{3}$ Institute for Cosmic Ray Research, University of Tokyo, Kashiwa, Chiba 277-8582, Japan.

Department of Theoretical Physics, University Autonoma Madrid, 28049

${ }^{6}$ Department of Physics, Boston University, Boston, MA 02215, USA.
Dept of Physics, British Columbia Institute of Technology, Burnaby, BC, V5G 3H2, Canad

${ }^{7}$ Department of Physics, British Columbia Institute of Technology, Burnaby, BC, V5G 3H2, Canada.

${ }^{8}$ Department of Physics and Astronomy, University of California, Irvine, Irvine, CA 92697-4575,
${ }^{7}$ Department of Physics, California State University, Dominguez Hills, Carson, CA 90747, USA.

${ }^{8}$ Department of Physics, California State University, Dominguez Hills, Carson, CA 90747, USA.
${ }^{9}$ Institute for Universe and Elementary Particles, Chonnam National University, Gwangju 61186, Korea.

${ }^{9}$ Institute for Universe and Elementary Particles, Chonnam National U
${ }^{10}$ Department of Physics, Duke University, Durham NC 27708, USA.

${ }^{11}$ Ecole Polytechnique, IN2P3-CNRS, Laboratoire Leprince-Ringuet, F-91120 Palaiseau, France.

${ }^{12}$ Junior College, Fukuoka Institute of Technology, Fukuoka, Fukuoka 811-0295, Japan.

${ }^{3}$ Department of Physics, Gifu University, Gifu, Gifu 501-1193, Japan.

${ }^{14}$ GIST College, Gwangju Institute of Science and Technology, Gwangju 500-712, Korea.

${ }^{5}$ Department of Physics and Astronomy University of Hawai, Honolulu, HI 96822 , USA

${ }^{16}$ Institute For Interdisciplinary Research in Science and Education, ICISE, Quy Nhon, 55121, Vietnam.

${ }^{17}$ Department of Physics, Imperial College London, London, SW7 2AZ, United Kingdom

8 Dipartimento Interuniversitario di Fisica, INFN Sezione di Bari and Università e Politecnico di Bari, I-70125, Bari, Italy.

${ }^{19}$ Dipartimento di Fisica, INFN Sezione di Napoli and Università di Napoli, I-80126, Napoli, Italy.

${ }^{20}$ Dipartimento di Fisica, INFN Sezione di Padova and Università di Padova, I-35131, Padova, Italy.

${ }^{21}$ INFN Sezione di Roma and Università di Roma "La Sapienza", I-00185, Roma, Italy.

${ }^{22}$ Department of Physics, Keio University, Yokohama, Kanagawa, 223-8522, Japan.

${ }^{24}$ Hepartment of Physics, King's College London, London, Tsukuba, Ibaraki

${ }^{25}$ Department of Physics, King's College London, London, WC2R 2LS, UK
${ }^{2}$ of Physics, Kobe University, Kobe, Hyogo 657-8501, Japan.

${ }^{25}$ Department of Physics, Kobe University, Kobe, Hyogo 657-8501, Japan.

${ }^{27}$ Department of Physics, Kyoto University, Kyoto, Kyoto 606-8502, Japan.

${ }^{28}$ Department of Physics, Miyagi University of Education, Sendai, Miyagi 980-0845, Japan.

${ }^{29}$ Institute for Space-Earth Environmental Research, Nagoya University, Nagoya, Aichi 464-8602, Japan.

${ }^{30}$ Kobayashi-Maskawa Institute for the Origin of Particles and the Universe, Nagoya University, Nagoya, Aichi 464-8602, Japan.

${ }^{31}$ National Centre For Nuclear Research, 02-093 Warsaw, Poland.

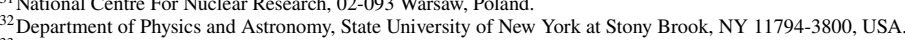

${ }^{33}$ Department of Physics and Astronomy, State University of New York at Stony Brook,
${ }^{3}$ Department of Physics, Okayama University, Okayama, Okayama 700-8530, Japan

${ }^{34}$ Department of Physics, Oxford University, Oxford, OX1 3PU, United Kingdom.

${ }^{35}$ Rutherford Appleton Laboratory, Harwell, Oxford, OX11 0QX, UK.
Rutics

${ }^{36}$ Department of Physics, Seoul National University, Seoul 151-742, Korea.

${ }^{37}$ Department of Physics, Seoul National University, Seoul 151-742, Korea.
${ }^{3}$ Physics and Astronomy, University of Sheffield, S3 7RH, Sheffield, United Kingdom.

${ }^{38}$ Department of Informatics in Social Welfare, Shizuoka University of Welfare, Yaizu, Shizuoka, 425-8611, Japan.

${ }^{38}$ STFC, Rutherford Appleton Laboratory, Harwell Oxford, and Daresbury Laboratory, Warrington, OX11 0QX, United Kingdom.

${ }^{39}$ STFC, Rutherford Appleton Laboratory, Harwell Oxford, and Daresbury La
${ }^{40}$ Department of Physics, Sungkyunkwan University, Suwon 440-746, Korea.

${ }^{41}$ Department of Physics, Faculty of Science, Tohoku University, Sendai, Miyagi, 980-8578, Japan.

${ }^{42}$ Department of Physics, Tokai University, Hiratsuka, Kanagawa 259-1292, Japan.

${ }^{43}$ The University of Tokyo, Bunkyo, Tokyo 113-0033, Japan.

${ }^{4}$ Department of Physics, University of Tokyo, Bunkyo, Tokyo 113-0033, Japan.

${ }^{5}$ Kavli Institute for the Physics and Mathematics of the Universe (WPI), The University of Tokyo Institutes for Advanced Study, University of Tokyo, Kashiwa, Chiba 277-8583, Japan.

${ }^{46}$ Department of Physics, Tokyo Institute of Technology, Meguro, Tokyo 152-8551, Japan.

${ }^{47}$ Department of Physics, Faculty of Science and Technology, Tokyo University of Science, Noda, Chiba 278-8510, Japan.

${ }^{48}$ Department of Physics, University of Toronto, ON, M5S 1A7, Canada.

${ }^{9}$ TRIUMF, 4004 Wesbrook Mall, Vancouver, BC, V6T2A3, Canad

${ }^{0}$ Department of Engineering Physics, Tsinghua University, Beijing, 100084, China.

${ }^{11}$ Faculty of Physics, University of Warsaw, Warsaw, 02-093, Poland.

${ }_{53}$ Department of Physics, University of Warwick, Coventry, CV4 7AL, UK

${ }^{54}$ Department of Physics, Yokohama National University, Yokohama, Kanagawa, 240-8501, Japan.

${ }^{4}$ also at BMCC/CUNY, Science Department, New York, New York, 1007, USA.

${ }^{5}$ Deceased. 